Techniques \& Culture

\title{
Doudous obsolètes?
}

Obsolete teddy-bears?

Frédéric Joulian

\section{(2) OpenEdition \\ Journals}

Édition électronique

URL : https://journals.openedition.org/tc/6217

DOI : $10.4000 /$ tc. 6217

ISBN : 1952-420X

ISSN : 1952-420X

Éditeur

Éditions de l'EHESS

Édition imprimée

Date de publication : 15 juin 2012

Pagination : 6-11

ISBN : 2-7351-1512-7

ISSN : 0248-6016

\section{Référence électronique}

Frédéric Joulian, «Doudous obsolètes ? », Techniques \& Culture [En ligne], 58 | 2012, mis en ligne le 04 décembre 2012, consulté le 29 septembre 2022. URL : http://journals.openedition.org/tc/6217 ; DOI : https://doi.org/10.4000/tc.6217 


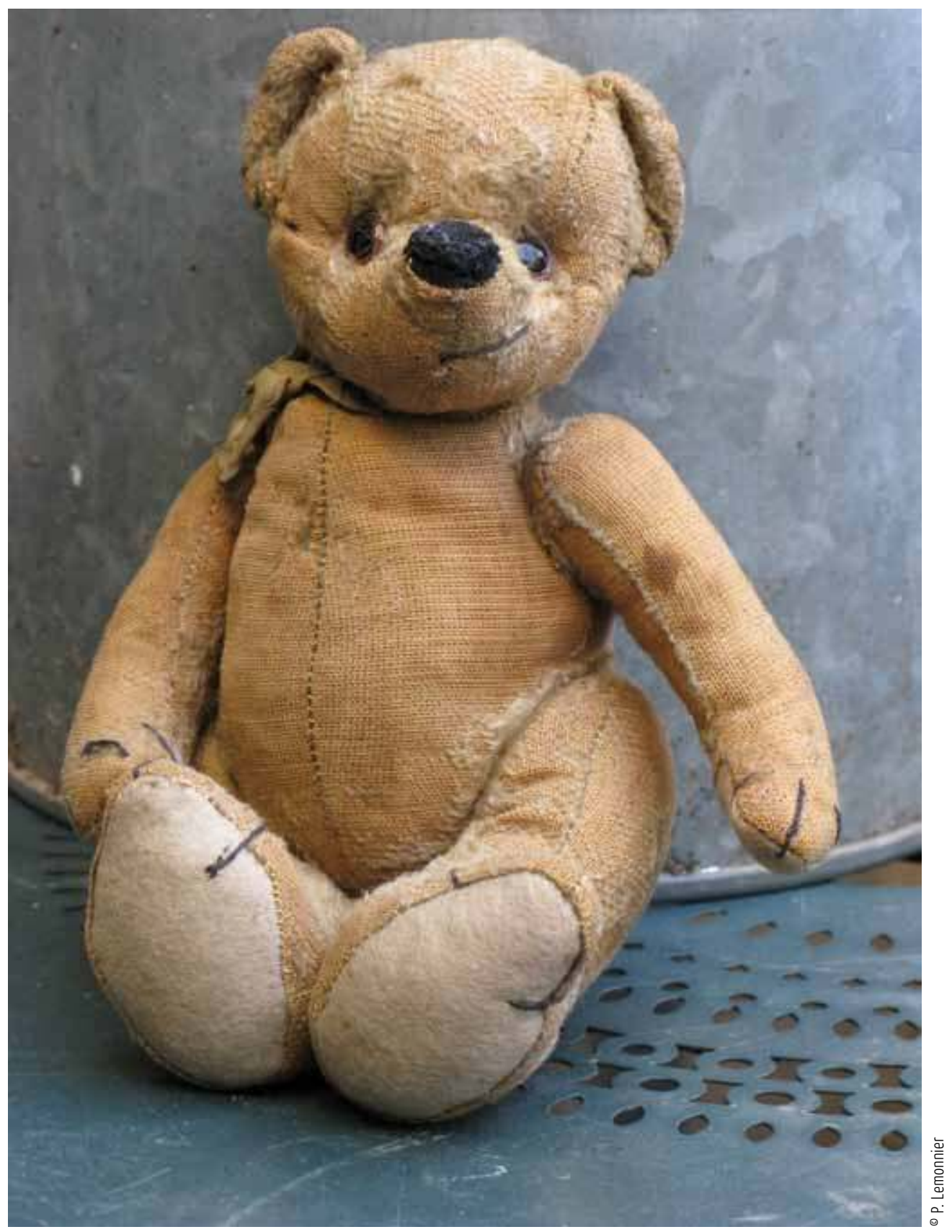




\section{DOUDOUS OBSOLÈTES?}

Avec ce nouveau volume de Techniques\&culture le lecteur a en main un objet original, fruit du travail d'édition de Sandra Revolon et Pierre Lemonnier, deux ethnologues océanistes, et de Maxence Bailly, archéologue protohistorien, qui ont réuni-car telle est notre formule interdisciplinaire et thématique depuis cinq ans maintenant-archéologues, historiens, ethnologues autour de la notion « d'irremplaçabilité » et dont nous dirons immédiatement la relativité dès lors qu'elle s'applique aux usages sociaux et culturels des objets. Ce volume fait suite à un numéro sur le Japon (T\&C n 57 ) lui aussi orienté « objet » et à diverses publications et entreprises collectives récentes d'exemplarisation ou de torpillage desdits objets (Debary, Turgeon 2007; Houdart, Thiery 2011; Watteau 2011 ; Anstett, Gélard 2012). Si l'objet est aujourd'hui à la mode ce n'est pas en raison d'un héritage barthésien un peu mou mais parce que l'on perçoit plus nettement combien les artéfacts sont trompeurs, d'apparence simple et de statut compliqué.

Le « retour de l'objet » sur le devant de la scène anthropologique se doit donc d'être interrogé à nouveaux frais et l'amateur s'étonnera peut-être de voir dans notre revue -lieu de promotion de l'analyse des gestes, systèmes, modes de production et savoir faire autrement dit, lieu d'analyse des « objets en action » plus que des « objets signes »-, un tel revival; mais ce serait à tort. C'est au contraire parce que le temps a passé et que nous avons réconcilié les modes de faire et de penser, la production et la consommation, les significations du faire et celles de l'être, que les objets peuvent être abordés sans ambages. Non que le dualisme ou l'ontologie de l'objet n'affectent pas toujours nos façons de poser 


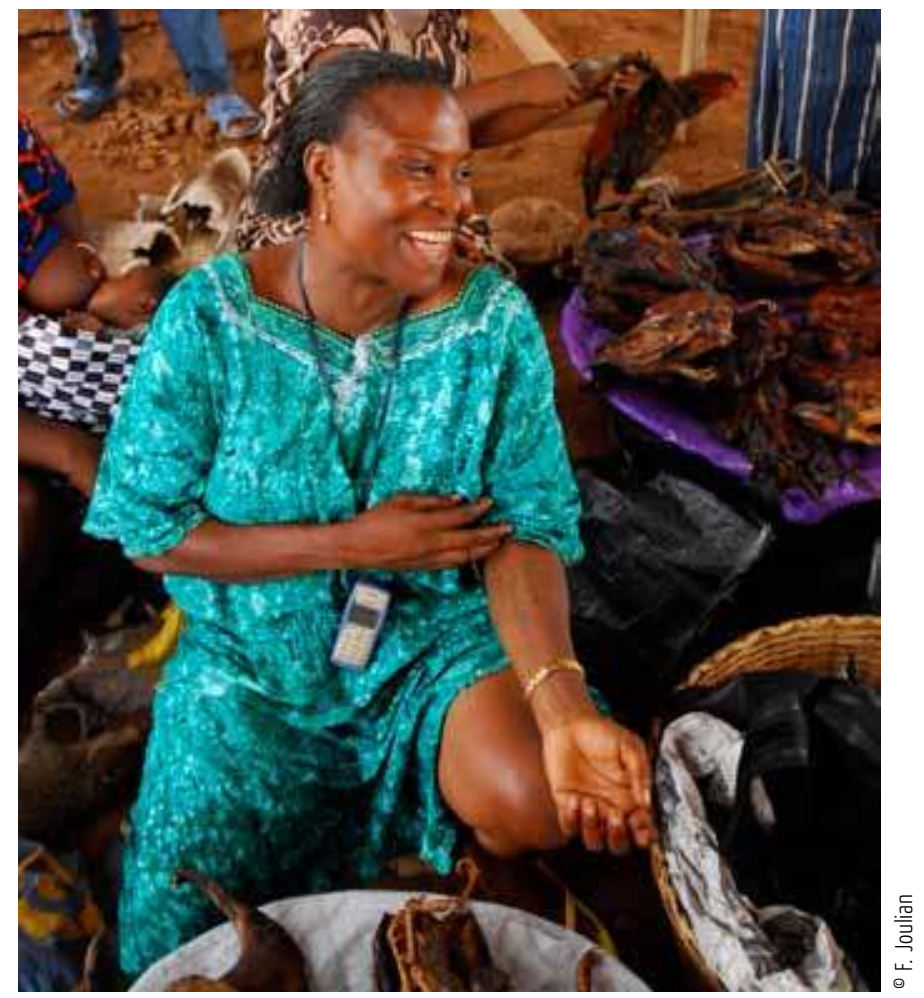

les problèmes, mais ici, ces objets irremplaçables sont pris comme viatiques ou exempla de configurations ou relations sociales originales à décrypter et restituer.

Au-delà de l'idée même d'irremplaçabilité, ce numéro s'ouvre sur une image: un nounours soixantenaire, vu de dos, usé jusqu'à la trame répondant au petit nom de « Ninisse». Il permet d'évoquer la question de l'irremplaçable à un niveau individuel, celui des premiers moments sensibles des expériences humaines, domaine plus habituel des psychologues que des anthropologues. Cet objet dit « transitionnel » (Winnicott 1973), joue le rôle de transducteur dans lequel il aide au développement psychologique et identitaire de l'enfant. Il est censé permettre une autonomisation vis-à-vis de la mère et l'accès aux autres. Mais le doudou n'est bien évidemment pas qu'individuel et, dès les premiers moments de vie humaine, les contextes sociaux et culturels jouent à plein. On n'imaginera guère un père ou une mère dans un jardin public dire " prête ton doudou à ton petit camarade » mais plutôt « prête ta pelle ou ton seau ». Ces objets intimes, durables et irremplaçables, analogues mais tous différents, dotés de

Grace, vendeuse de viande de brousse sur le marché de Ho au Ghana, utilise son cellulaire pour ajuster ses déplacements et rendez-vous avec les chasseurs et clients. Au second plan à gauche l'objet transitionnel n'est pas encore nécessaire, 2007. leurs textures, odeurs, formes propres, contrastent fortement avec les objets obsolescents de nos univers contemporains, objets programmés pour ne durer qu'un temps limité et obéissant aux cycles courts et surproductifs de nos économies irresponsables. Ce contraste entre deux relations aux objets illustre peut-être davantage nos affects et conceptions que ne le font nos discours si loquaces en la matière. Qu'est-ce qu'un objet, qu'un bien précieux et irremplaçable dans une petite société traditionnelle ou dans une société moderne mondialisée? La question de l'originalité, de la préciosité ne se posera bien évidemment pas de la même façon dans un univers artisanal, à produits et biographies uniques que dans un univers à codage et production de masse. Aujourd'hui, dans certains cas même, les objets sont équivalents à des codes numériques, entièrement algorithmisés et pouvant être générés n'importe où, n'importe quand, à l'aide d'imprimantes 3 D (Buchli 2009). Cette question de la reproductibilité illimitée de l'objet s'étend à présent aux différentes techniques de production du vivant, et au premier chef, au clonage, matérialisé depuis la brebis Dolly; artéfact, biofact, les limites se déplacent depuis le néolithique et l'apparition de la domestication.

Avec cette reproductibilité à l'identique et donc d'une « remplaçabilité » généralisée, nous pouvons évoquer aussi la question de la valeur marchande, qu'elle soit cauri, monnaie, billet, indice, ... et de l'échange, autrement dit des objets substituts de valeurs permettant le fonctionnement économique et symbolique des sociétés. Mais, ainsi que le montrent plusieurs articles de ce Thema, la valeur et ses représentations ne s'opposent pas forcément à l'objet unique qui peut lui-même être valeur ou s'inscrire dans des systèmes permettant la continuité.

Remarquons par ailleurs combien l'anthropologie de la culture matérielle contemporaine, comme celle de la technologie culturelle qui l'a précédée, ne s'intéresse ni aux objets pour eux-mêmes, ni aux significations sociales et culturelles des objets et 
techniques, mais bel et bien aux effets particuliers des matériaux, objets et techniques sur les groupes et relations sociales. Ce point de vue " en production » et " en réception », pourrait-on dire, se trouve porté dans ce Thema autant par les anthropologues que par les historiens ou les archéologues.

Les objets irremplaçables sont également essentiels à la survie, au fonctionnement du groupe, ils sont en deçà des significations fonctionnelles ou genrées, et ce même s'ils ont un sens unique pour un individu (car ils s'attachent à des biographies particulières, à une main et non à toutes les mains), ou un sens partagé pour une société entière. Il convient aussi de bien définir les fonctions de ces objets. Du simple canif dans la poche de tout homme de la campagne il y a quelques décennies, au téléphone cellulaire de l'urbain d'aujourd'hui, on perçoit les mutations majeures des fonctions primaires, de motrices à communicantes. Les univers quotidiens sont désormais surpeuplés d'objets à communiquer et à s'évader. À la différence des objets de la génération précédente, la radio et la télévision, encore attachés au foyer et à un lieu, les petits objets mobiles génèrent des usages, des socialisations, des confrontations de domaines (intimes/publics avec les téléphones portables; travail/privé avec les nouvelles tablettes mi-ordinateur, mi-téléphone) que nous peinons encore à décrire d'un point de vue culturel. Génèrent-ils l'uniformisation des conduites à un niveau planétaire ou bien l'appropriation et la variabilité dans la différence culturelle ou a-culturelle (Ito \& al. 2005; Dant 2008)? De vastes chantiers ethnographiques s'ouvrent à nous.

Si nous nous déplaçons à présent du côté des images médiatisées, force est de constater la puissance des représentations qui donnent à percevoir de nouveaux objets, auparavant invisibles (nano ou macro, mais aussi calculés, numériques) dont les effets cognitifs sont encore difficilement appréciables. L'objet « Terre », visible depuis les satellites et les missions Appolo de 1968, s'appréhende dans son entièreté. Elle est tous les jours sous nos pieds et « vue du ciel », la Terre, support de toute vie, et au passage, de l'humanité, est depuis longtemps mise à mal par de multiples actions humaines vulnérantes mais en des lieux circonscrits. Aujourd'hui, avec la prise de distance, on peut voir le réchauffement climatique et ses effets (le trou dans la couche d'ozone notamment) et faire comprendre combien nous sommes tous liés à un même destin rapide, à des événements qui nous affectent tous. S'apitoyer autrefois sur le sort du Biafra n'engageait que notre empathie, nos questionnements moraux. Voir les effets des tsunamis ou typhons actuels nous touche directement; nous sommes tous sur la même planète dont nous percevons les limites. Les effets papillons ont muté en effets ptérodactyles! Cette finitude de notre univers désormais représenté de loin, de l'espace, et dont on voit les changements néfastes au jour le jour, contraste si fort avec nos façons d'agir et de penser et avec nos cosmologies locales et immédiates. À l'inverse peut-être du territoire réduit des petites sociétés humaines auquel correspondent des univers invisibles, les sociétés modernes et leur science n'ouvrent qu'à un cosmos, distant, mais accessible. Mais ce n'est là qu'une suggestion d'éditorial! La partie Curiosa poursuit la veine de l'irremplaçabilité par deux propositions, la première de Yann-Philippe Tastevin, sur des objets déplacés ailleurs et réappropriés: les Ape, Tuk-tuk, Rickshaw,... voiturettes à trois roues produites en Inde et exportées dans le cadre d'échanges «Sud-Sud» qui concurrencent les modèles industriels automobiles dominants « Nord-Sud ». Dans la seconde, avancée par Sandra Revolon à propos d'un objet anthropologique improbable, l'iridescence, nous trouvons une entrée par les images

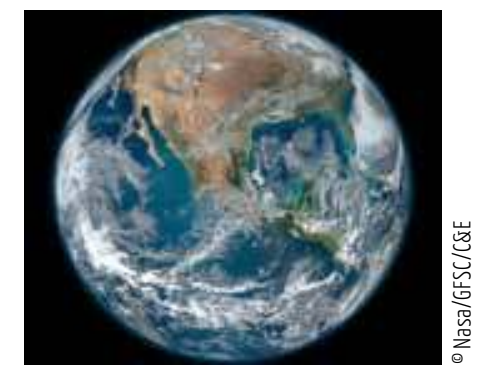

La terre vue du ciel, 2012 
qui nous donnent à penser ce que sont l'immatériel et les morts pour les Owa des Îles Salomon. On y voit comment des effets d'optique observables dans la nature sont captés par les hommes et transformés en objets conçus pour s'assurer la présence des défunts parmi eux. Certains d'entre nous se rappelleront alors la présence attentionnée, curieuse et savante d'Isac Chiva, disparu au printemps dernier et qui avait accompagné la reprise de la revue d'un article sur les patrimoines naturels et l'ethnologie de la Corse (Chiva 2008).

J'aimerais finalement évoquer les trois articles publiés en Varia. Le premier porte sur des objets et des phénomènes de mode (Benfoughal) qui analyse les vanneries contemporaines du Maghreb faites à partir de sacs plastiques et soulève de façon extrêmement bien documentée comment certaines traditions artisanales (fondées sur des savoir-faire originaux, des couleurs, des circuits de fabrication) intègrent de nouveaux matériaux en les recyclant dans une culture locale. Les deux autres articles abordent le travail temporaire et les reconfigurations qu'il opère sur la vie des personnes et en particulier sur les modes de transmissions. Dans les univers discontinus, à CDD généralisés dans le secteur tertiaire, il nous faut repenser les savoir-faire, l'identité professionnelle et individuelle et bien sûr le rapport au temps et aux projets de vie (Rosini). Cette question du temps est également au cœur de la recherche de L. Baghioni qui explore les logements et les huis clos générés par la précarité du travail saisonnier. Elle les décrit de façon très subtile comme des indicateurs de nos sociétés aux différences sociales de plus en plus accusées.

Ces deux dernières propositions émanent de jeunes chercheurs hors-statut et ne sont pas sans nous rappeler que nous sommes en période de crise économique. La revue vient de subir des coupes budgétaires drastiques. Nos institutions, CNRS en tête, nous poussent à uniformiser, à entrer dans le moule des publications électroniques des sciences dures, alors qu'à l'inverse, plus que jamais, nous pensons qu'il convient de maintenir la diversité des formes d'adresse au public, raison pour laquelle nous avons opté pour une revue-livre de qualité, objet reproductible, mais non remplaçable d'un clic d'écran.

« Doudou avec tétines», perdu sur un parking de La Valette du Var, juin 2012.

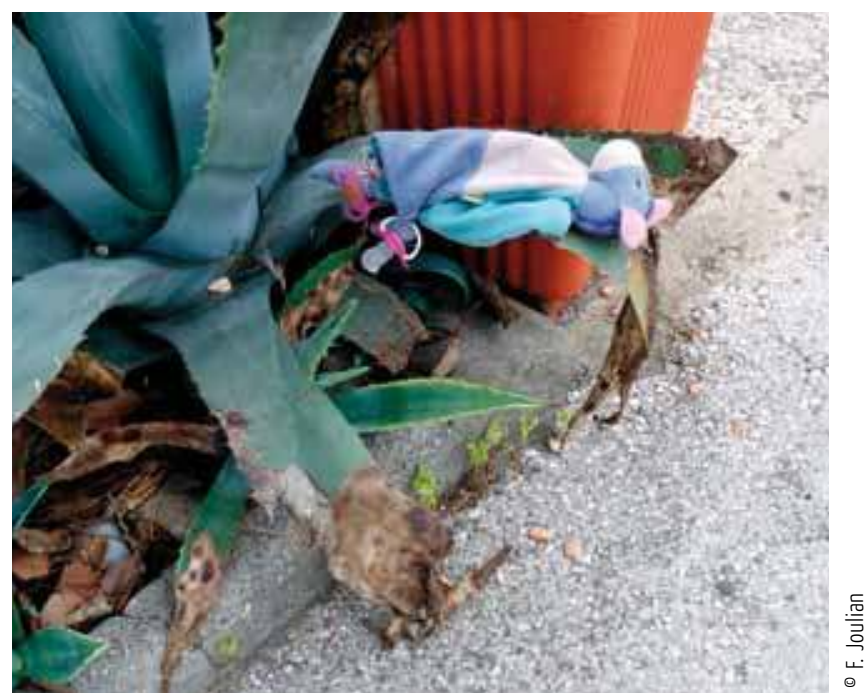




\section{NOTES}

Photo d'ouverture: Ninisse, 1950.

\section{RÉFÉRENCES}

Anstett, E., Gélard, M.-L. 2012 Les Objets ont-ils un genre? Culture matérielle et production des identités sexuées. Paris: Armand Colin, 244 p.

Buchli, V. 2009 La Culture matérielle, la numérisation et le problème de l'artéfact. In TechniquesEculture n 52-53 Technologies (dir.) L. Coupaye, L. Douny: 212-231.

Chiva, I. 2008 Sciences de l'homme et patrimoines naturels. Quelques jalons historiques et théoriques. In TechniquesEculture $\mathrm{N}^{\circ} 50$ Les Natures de l'Homme (dir.) S. de Cheveigné, F. Joulian: 16-39.

Dant, T. 2008 iPod... iCon. In Italian in Studi Culturali, Vol. 5 (3): 355-373.

Debary, O., Turgeon, L. (dir.) 2007 Objets et mémoires. Paris, Québec: Éditions de la MSH, Presses de l'Université Laval, $251 \mathrm{p}$.

Houdart, S., Thiery, O. 2011 Humains, non humains. Comment repeupler les sciences sociales? Paris: La Découverte, $368 \mathrm{p}$.

Ito, M., Okabe, D., Matsuda, M. (ed.) 2005 Personal, portable, pedestrian: Mobile phones in Japanese life. Cambridge: MIT Press.

Watteau, F. (ed.) 2011 Profils d'objets. Approches d'anthropologues et d'archéologues. Paris: De Boccard, 316 p. Winnicott, D. W. 1975 Jeu et réalité. L'espace potentiel. Paris: Gallimard, 213 p. 